\title{
HIGH SPEED MEMORY EFFICIENT EBCOT ARCHITECTURE FOR JPEG2000
}

\author{
Hung-Chi Fang, Tu-Chih Wang, Chung-Jr Lian, Te-Hao Chang and Liang-Gee Chen \\ DSP/IC Design Lab \\ Graduate Institute of Electronics Engineering and Department of Electrical Engineering \\ National Taiwan University \\ 1, Sec. 4, Roosevelt Rd., Taipei 106, Taiwan \\ \{honchi, eric, cjlian, thchang, Igchen\}@video.ee.ntu.edu.tw
}

\begin{abstract}
This paper presents a high speed, memory efficient architecture of embedded block coding with optimized truncation (EBCOT) tier- 1 in JPEG2000. By parallel coding all the bitplanes, the state variable memory can be eliminated. The proposed architecture can process $50 \mathrm{M}$ coefficients per second at $100 \mathrm{MHz}$, which can realtime encode $720 \mathrm{p}$ resolution of HDTV picture format at $30 \mathrm{fps}$.
\end{abstract}

\section{INTRODUCTION}

JPEG2000 [1], [2] is the latest standard for still image coding. It is well known by its excellent coding performance and abundant features [3], such as region of interest (ROI), scalability, error resilience, etc. Although these features can also be provided in JPEG standard, some functionalities require supports at decoder side. On the other hand, all these powerful tools can be provided in a single JPEG2000 bitstream. For example, an image can be coded in lossless mode for storage and be retrieved at different bitrates. However, the high computational complexity that grants such excellent performance and rich features restricts the real time applications of JPEG2000.

EBCOT is the most complicated part in JPEG2000 [4]. There are various architectures of EBCOT proposed in previous arts [4], [5], [6], [7]. All of them process the block bitplane by bitplane, which is the default mode of JPEG2000 block coding. Although the compression ratio of the default mode is the highest, it has some drawbacks. First, the default mode performs bad at error resilience since the arithmetic encoder (AE) terminates at end of the code block only. When an error occurs somewhere in a code block all the rest data in the code block becomes useless. Second, the hardware implementation of the default mode requires needs a lot of memory to store the state variables. The memory requirement analysis in [8] shows that $20 K$ bits of internal memory are needed for the block coding with $64 \times 64$ code block size.

The discrete wavelet transform (DWT), adopted by JPEG2000, is a word-level processing. Since the block coding follows DWT and processes in a bitplane by bitplane manner, there should be some data conversion between these two parts. The block coder access one bit of the coefficient at one time. If the coefficient bitwidth is $n$, the subband memory will be accessed $n$ times and the power consumption will be large.

All these drawbacks can be solved with parallel processing. As shown in the following sections, all the memory for state variables can be avoided. The power consumption of memory access

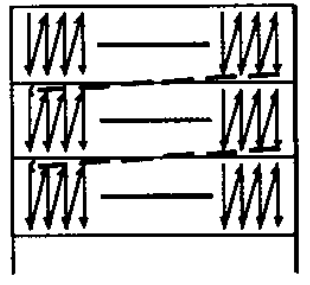

(a)

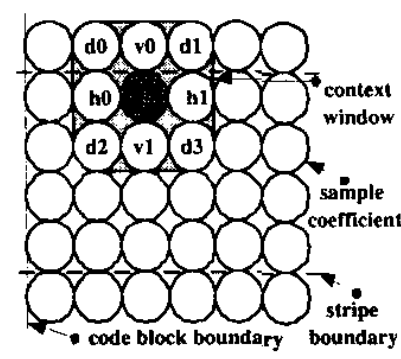

(b)
Figure 1: (a) Scan pattern for bitplane coding. (b) Context window for context formation.

can be reduced since the proposed block coder processes DWT coefficients at word-level as well as DWT. Error does not propagate across passes since $\mathrm{AE}$ terminates at each coding pass in parallel mode.

This paper is organized as follows. Section 2 reviews the EBCOT algorithm. The proposed EBCOT architecture is presented at section 3. Implementation result and comparisons are shown at section 4 . Section 5 gives a brief conclusion of this paper.

\section{EBCOT ALGORITHM}

JPEG2000 adopts EBCOT coding as its entropy coding algorithm. The basic unit of EBCOT coding is a rectangle called code block. Its size is typically $64 \times 64$ or $32 \times 32$. A code block is further divided into stripes that are $4 \times N$ rectangles, where $N$ represents code block width. The scan order is first column by column within a stripe and then row by row for stripes as shown in Fig. 1(a). The context of a sample coefficient is formed according to the significant state of itself and its eight neighbors within a $3 \times 3$ window, as shown in Fig. 1(b)

The order of bitplane coding is from the MSB to the LSB. Each bitplane contains three coding passes called significant propagation pass (pass 1), magnitude refinement pass (pass 2 ) and clean up pass (pass 3). The exception is the MSB bitplane of the code block, which has only one pass (pass 3 ). By examining the significant states of the nine sample coefficients in the context window, the coding pass of the central sample coefficient can be decided. The context is generated from the significant state of its eight neighbors, each labelled as h0, h1, etc. in Fig. 1(b). These 


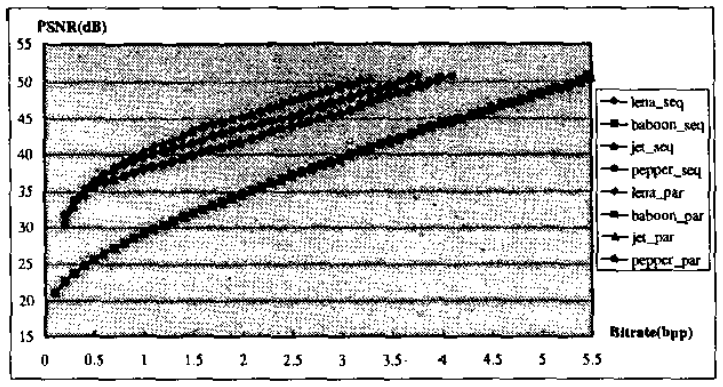

Figure 2: Performance comparison of default and parallel mode.

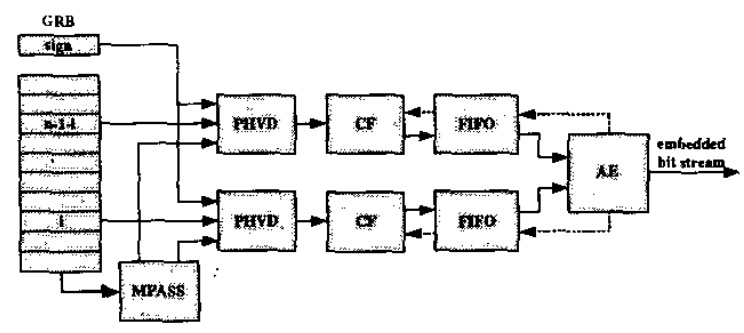

Figure 3: Architecture of Paraliel EBCOT.

eight values are called HVD for a short hereafter. Finally, AE processes the context and decision pair and outputs the bitstream segment.

There are mainly two differences between the default and parallel EBCOT coding algorithms. First, AE should make termination at the end of every coding pass in parallel mode. By doing so, each pass of the same bitplane can be coded in parallel. Second, the sample coefficients that come from the lower stripe are considered as insignificant in parallel mode.

The performances of these two mode are almost the same as shown in Fig. 2. The images are of size $512 \times 512$ and are coded with 53 filter. The average PSNR degradations for 53 and 97 filters are $0.17 \mathrm{~dB}$ and $0.19 \mathrm{~dB}$ respectively. Although the performance of the parallel mode is slightly poorer than the default mode, the parallel mode has some advantages. It is more robust in errorprone environment than the default mode since $\mathrm{AE}$ terminates at the end of every pass. When an error occurs, it influences only the rest data in the same pass rather than the whole code block in the default mode. And it requires only one scan to code the three pass, while default mode needs three scans. In addition, it is much easier for tier-2 implementation, which makes optimized truncation by terminating $\mathrm{AE}$ at each pass.

\section{PARALLEL EBCOT ARCHITECTURE}

The proposed high speed and memory efficient parallel EBCOT architecture is shown in Fig. 3. There are five major functional blocks in our design. All of them process independently over bitplanes except the MPASS module. The subband data after DWT is fed into MPASS where the coding pass number of the MSB bitplane is calculated. Then, the pass and HVD data of all bitplanes

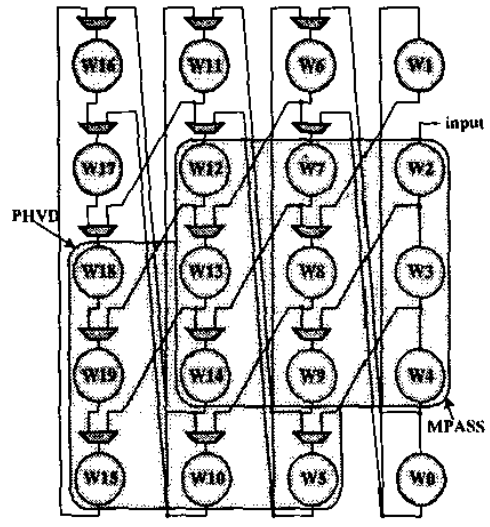

Figure 4: Gobang Register Bank. The circles represent registers that contains input data.

are determined in the PHVD module. The CF module maps the pass and HVD data into context-decision pairs and passes them to the FIFO. Each AE handles two FIFO inputs and generates output bitstreams.

The number of $\mathrm{AE}$ can be folded by two due to the properties of DWT coefficients in EBCOT algorithm. The magnitudes of coefficients after DWT are not equal distributed and large coefficients are much less then small one. And the number of contexts in a bitplane decreases dramatically from LSB to MSB. By these observations, we use one AE to deal with two bitplanes. Bitplane $i$ and Bitplane $(n-1-i)$ use the same AE to encode bitstream, which makes the hardware cost of $A E$ half. The number $n$ represents the number of bitplane supported by the proposed architecture.

\subsection{Gobang Register Bank}

The input data is first stored and shifted in the Gobang Register Bank (GRB), as shown in Fig. 4. The GRB reorder the input data to other modules to meet JPEG2000 scan order. The data is first rotated within each column to match the data flow of one column in the stripe. When a column in the stripe is coded, i.e. every four clock cycles, the data sample is shifted to next column for the next column in the stripe. The two $3 \times 3$ windows, MPASS and PHVD, indicate which registers are used in the MPASS and PHVD module. The GRB contains 20 word-level registers instead of 24 registers since the sample coefficients from next stripe are considered zero.

\subsection{MPASS}

The major difference between parallel mode and default mode implementations is the number of scans. Our parallel architecture requires only one scan instead of three in the default mode. A problem of determining significant state accurs when processing three passes in one scan. Fig. 5 shows an example of this problem. The meanings of circles are the same as those in Fig. 1(b). The value in circle represents the coefficient value. A gray circle represents that the coefficient has been significant in upper bitplane while a white circle means not significant. Referring to the truth table in this example, the contribution of coefficient at position $\mathrm{v} 1$ 


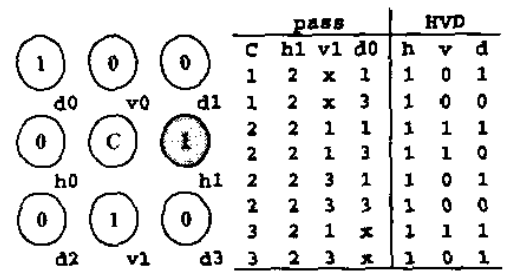

Figure 5: Example of relationship between coding pass and significant contribution.

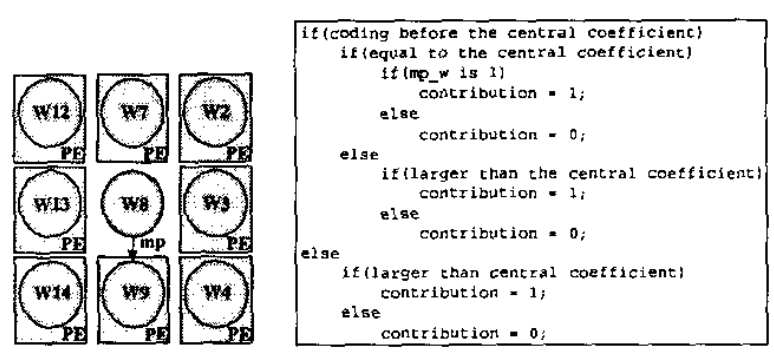

Figure 6: Block diagram and pseudo-code of MPASS module.

is inffuenced by the coding pass it belongs. As a result we must have the pass information of all the neighbors in order to calculate the correct HVD data. However, we need not to get the pass information of every bitplane. Because the significant state is always 1 in bitplane lower than MSB and is always 0 in bitplane higher than MSB regardless of the coding pass. The MSB is only one bitplane that the updated significant state differs to the one before update.

The MPASS module is designed to solve this problem. The block diagram of MPASS is shown in Fig. 6. The circles represent the registers in GRB. Each square represents a processing element (PE) and the pseudo-code is shown in Fig. 6. The coding pass of the MSB of the central coefficient is called "mp" and is merged into the data flow with the coefficient in GRB. The signal "mp_w" is the mp of the neighboring coefficient since it is coded before the central coefficient. If all the contributions of eight PEs are zero, $\mathrm{mp}$ of the central coefficient is zero; otherwise, it is one.

\subsection{PHVD}

PHVD module determines the coding pass and calculates the HVD values to form the context. The block diagram of PHVD and the truth table of the PEs are shown in Fig. 7. The mps and $\mathrm{mp}$ indicate the coding pass of MSB of the central and neighboring coefficients. The input "fo" indicates whether the bitplane is the MSB. The input "sig" indicates if the bitplane is lower than MSB. The input "cnt" is the counter corresponding to the position of the central coefficient in the stripe. When cnt is zero, the central coefficient in MPASS module is the first pixel in a column. Due to pipeline, the central coefficient in PHVD is the first coefficient in the stripe when cnt is 1 . The outputs of the PEs are call contribution (cont). Both the coding pass and HVD values are determined by the contribution of these neighbors. There are three kinds of PEs in PHVD which calculate the contribution of the corresponding sample coefficient. PE0 is responsible for the neighbor which is coded after

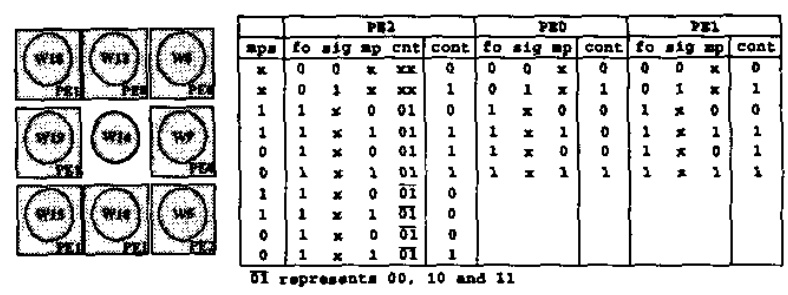

Figure 7: Block diagram of PHVD module.

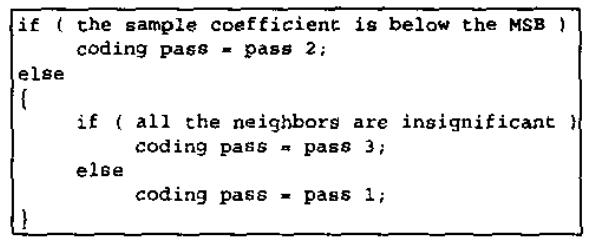

Figure 8: Pseudo code which determines the coding pass.

the central coefficient. And PE1 is responsible for the neighbor which is coded before the central coefficient. The coefficient corresponding to PE2 is a special case. It comes from the previous stripe when cnt is 1 and from the same stripe at other cases of cnt. So it is coded before the central coefficient when cnt equals 1 but after the central coefficient at other cases. The pseudo code which determines the coding pass of the central coefficient is shown in Fig. 8. The outputs of the PEs are grouped as horizontal $(\mathrm{H})$, vertical (V) and diagonal (D) as the context window in Fig. 1(b). And the HVD data is calculated by adding the contributions in the same group. Note that the PHVD window is not the same as in MPASS since all the coefficients in the PHVD window must go through MPASS module.

\subsection{Context Formation}

The block diagram of context formation module is shown in Fig. 9. The coeff indicates the four sample coefficients in the same column of a stripe. Run_length is an flag indicating whether the column should be coded in run-length mode or not. Sigma is also a flag representing the first magnitude refinement. Band indicates which subband the code block belongs. Ctxd_s is the context and decision of the sign of the sample coefficient.

The CF module works as follows. First, pass and HVD data come from previous module, PHVD, are used to check the context of the sample coefficient. The $\mathrm{R}$ module is a register. The $\mathrm{ZC}$ and MR module map the pass and HVD data into zero coding and magnitude refinement. In order to cope with the run-length code, we must keep the first three contexts in a column of stripe. Finally, the contexts and decisions are generated by the run length code (RLC) module. Note that there are variable number of contexts generated, from 0 to 4 , when coding a sample coefficient.

\subsection{FIFO}

Because the output rate of CF module is not identical with that of $\mathrm{AE}$, we need a fifo between these two modules. The length 


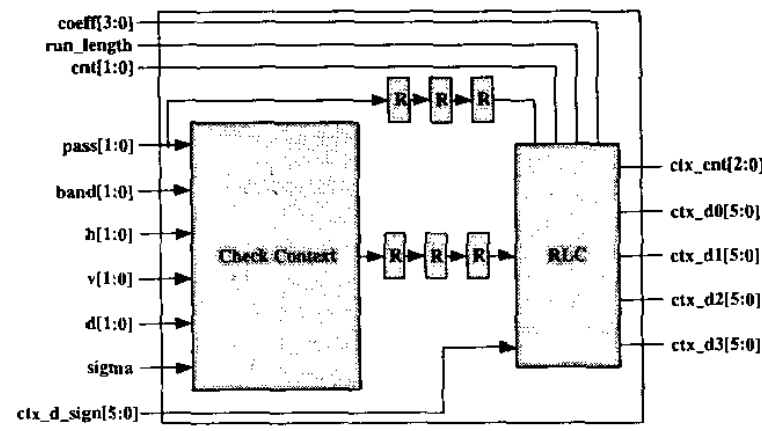

Figure 9: Block diagram of context formation module.

of fifo is chosen as fifteen, experimentally. Each fifo contains a pass ( 2 bits) and four context-decision pair (each 5 bits). Because the coding pass is indicated explicitly, the number of bit used to represent context is reduced to 4 bits. 10 FIFOs are used since there are 10 magnitude bitplanes in our architecture.

\subsection{Arithmetic Encoder}

Although one sample coefficients belongs to only one of three passes, we must have three sets of probability estimation table and other state variables in the $\mathrm{AE}$. The memory requirement is double since we have two bitplane that use a common AE. Note that the probability estimation tables are separated by coding passes and some context that will not appear in the certain coding pass can be eliminated. For example, run-length context will not appear in pass 1 and pass 2 and should be removed from the tables. By doing so, the area of AE can be further reduced.

\section{IMPLEMENTATION RESULT AND COMPARISONS}

\subsection{Implementation}

The proposed architecture is synthesized using TSMC $0.35 \mu \mathrm{m}$ CMOS technology. The gate count and memory requirement of our proposed architecture are listed in Table 1 . The size of the code block is $64 \times 64$ and the coefficients bitwidth is 11 bits.

Table 1: Hardware Requirement of the Proposed Architecture.

\begin{tabular}{ccc} 
& Datapath (gates) & Memory (bits) \\
\hline CF & 14803 & $64 \times 12$ \\
FIFO & NA & $(15 \times 22) \times 10$ \\
AE & $11536 \times 5$ & $(72 \times 6) \times 5$ \\
\hline
\end{tabular}

\subsection{Comparisons}

Table 2 lists the processing time of a code block. The number $\mathrm{N}$ represents the width of the code block and $\mathrm{n}$ represents the number of bitplanes. As we can see, the number of speed-up is about $n$ than Chen's and Chiang's and is almost $3 n$ than Taubman's if the number of non-zero bitplane is less than 6 . And when the number of non-zero bitplanes is lager than 6 , the speed up is half. Besides, the processing time of the proposed architecture is not proportional to the number of bitplanes.

Table 2: Processing Time Comparison with Other Architectures

\begin{tabular}{cc}
\hline & average processing time \\
\hline$[9]$ 's & $(3 \times(\mathrm{n}-1)+1) \times \mathrm{N}^{2}$ \\
[4]'s & $1.3 \times \mathrm{n} \times \mathrm{N}^{2}$ \\
[7]'s & $\mathrm{n} \times \mathrm{N}^{2}$ \\
proposed & $(1+\delta) \times \mathrm{N}^{2}$ \\
\hline
\end{tabular}

$\delta$ is about 0.1 to 0.2 when the nonzero bitplanes are smaller than or equal to 5 and close to 1 when larger than 5 .

\section{CONCLUSIONS}

In this paper, a high performance, memory-saving parallel architecture of EBCOT tier-1 for JPEG2000 is proposed. All the bitplanes are coded in parallel in our architecture. The state variable memory can be eliminated by the proposed method. And the processing rate is several times then other architectures previously proposed in the literature. The proposed architecture processes $50 \mathrm{M}$ coefficients per second at $100 \mathrm{MHz}$, regardless of the bitwidth of the coefficients, and can encode lossless HDTV 720p resolution pictures at $30 \mathrm{fps}$ in real time.

\section{REFERENCES}

[1] ISO/IEC JTC1/SC29/WG1 N1855, JPEG 2000 Part l: Final Draft International Standard (ISO/IEC FDIS15444-1), Aug. 2000.

[2] ISO/IEC JTC1/SC29/WG1 N1271, JPEG2000 Requirements and Profiles, Mar. 1999.

[3] Skodras A., Christopoulos C., and Ebrahimi T., "The JPEG 2000 Still Image Compression Standard," IEEE Signal Processing Mag., vol. 18, no. 5, pp. 36-58, Sept. 2001.

[4] K. F. Chen, C. J. Lian, H. H. Chen, and L. G. Chen, "Analysis and Architecture Design of EBCOT for JPEG-2000," in IEEEE Int. Symp. Circuits and Systems (ISCAS 2001), 2001, pp. 765-768.

[5] Kishore Andra, Chaitali Chakrabarti, and Tinku Acharya, "A High Performance JPEG2000 Architecture," in IEEEE Int. Symp. Circuits and Systems (ISCAS 2002), 2002, pp. 765-768.

[6] Y. T. Hsiao, H. D. Lin, and C. W. Jen, "High-Speed Memory Saving Architecture for the Embedded Block Coding in JPEG2000," in IEEEE Int. Symp. Circuits and Systems (ISCAS 2002), 2002, pp. 133-136.

[7] J. S. Chiang, Y. S. Lin, and C. Y. Hsieh, "Efficient PassParallel for EBCOT in JPEG2000," in IEEEE Int. Symp. Circuits and Systems (ISCAS 2002), 2002, pp. 773-776.

[8] D. Taubman, E. Ordentlich, M. Weinberger, and G. Serourssi, "Embedded Block Coding in JPEG 2000," in Proc. IEEE Int. Conf. Image Processing (ICIP 2000), 2000, pp. 33-36.

[9] ISO/IEC JTC1/SC29/WG1 N1684, JPEG-2000 Verification Model 7.0 (Techinical Description), Apr. 2000. 